U.S. Department of the Interior U.S. Geological Survey

\section{Floods In South Dakota, Spring 1995}

Severe flooding occurred across South Dakota during the spring of 1995. Similar to the floods of 1993, high flows began during snowmelt and continued as spring rains moved into the area. Localized flooding resulted from heavy precipitation in the form of snow and rain that occurred during late March and April over much of the central and eastern parts of the State. Unusually heavy spring rains followed in late April and May, especially in the Black Hills area. Unlike the 1993 floods, which were confined mostly to the James, Vermillion, and Big Sioux River Basins, the severe 1995 flooding was Statewide.

\section{The Storms}

A narrow band of heavy snow resulted from storms along the northern border in late February and by early March, snow depths were about 20 inches in the northeastern part of the State. Following an early March storm, record low temperatures were equaled or exceeded at five cities in eastern South Dakota, including $-32^{\circ} \mathrm{F}$ at Aberdeen on March 8. A very rapid warmup that began on March 10 caused ice breakup on many streams. Heavy precipitation occurred over central and eastern South Dakota April 8-12 and April 18 producing as much as 6 to 7 inches of moisture in the form of snow and rain. As much as 50 to 60 inches of snow fell in the central part of the State during the two storms, causing extended electrical outages and significant losses of newborn livestock. During April 28-30, another storm tracked across the southern part of the State; southwestern South Dakota received 1 to 2 inches of rain, the Black Hills of western South Dakota received 3 to 8 inches of snow, and the south-central and southeastern parts of the State received up to an inch of rain.

There were unusually intense rains in the western and northern Black Hills May 6-10. The storm produced widespread rainfall of 3 to 6 inches in much of the Black Hills, with reports of 6 to 10 inches in the northern Black Hills and foothills. During May 7-10, 8.78 inches of precipitation was recorded at the U.S. Geological Survey (USGS) precipitation gage, Two Bit Gulch near Deadwood. The Two Bit Gulch gage recorded 13.43 inches in May. The rest of the State received 1 to 3 inches of rain May 7-10.

Slow moving, intense thunderstorms over Memorial Day weekend in southeast South Dakota produced severe weather (tornadoes and hail) and more than 4 inches of rain in some areas. All-time record precipitation during March 1 through May 31 occurred at some locations (table 1) that have 100 or more years of record. Precipitation amounts, compiled by the South Dakota State Climatologist for March 1 through May 31 for selected National Weather Service stations across the State, are shown in figure 1 .

\section{The Floods}

Flooding began in the Grand River and the upper James River basins when the snowpack began to melt in early to midMarch. Localized flooding during March and April also occurred at several locations because of severe backwater from ice jams. On March 14, ice jams caused flooding that resulted in evacuation of 19 families from Wakpala and about 200 people from Bullhead, communities located on the Standing Rock Indian Reservation in north-central South Dakota. Above-normal snow and rainfall during late March and April added to the flooding, especially in eastern South Dakota. Additional precipitation during late April and May caused record stage and flow on many streams Statewide (table 2).

Table 1. Record precipitation during March 1 through May 31 for selected gages with 100 or more years of record

\begin{tabular}{lcc}
\hline & \multicolumn{2}{c}{$\begin{array}{c}\text { March-May precipitation, in } \\
\text { inches }\end{array}$} \\
\cline { 2 - 3 } Location & $\mathbf{1 9 9 \mathbf { 1 } ^ { \mathbf { 1 } }}$ & $\begin{array}{c}\text { Previous } \\
\text { record } \\
\text { (year) }\end{array}$ \\
\hline Faulkton & 12.99 & $12.89(1991)$ \\
Highmore & 14.63 & $13.93(1991)$ \\
Huron & 14.96 & $12.80(1942)$ \\
Mitchell & 15.55 & $15.28(1942)$ \\
Sioux Falls & 14.64 & $13.78(1920)$ \\
Yankton & 16.94 & $15.89(1883)$ \\
\hline
\end{tabular}

${ }^{1}$ Preliminary National Weather Service data (A.R. Bender, State Climatologist, written commun., June 1995)

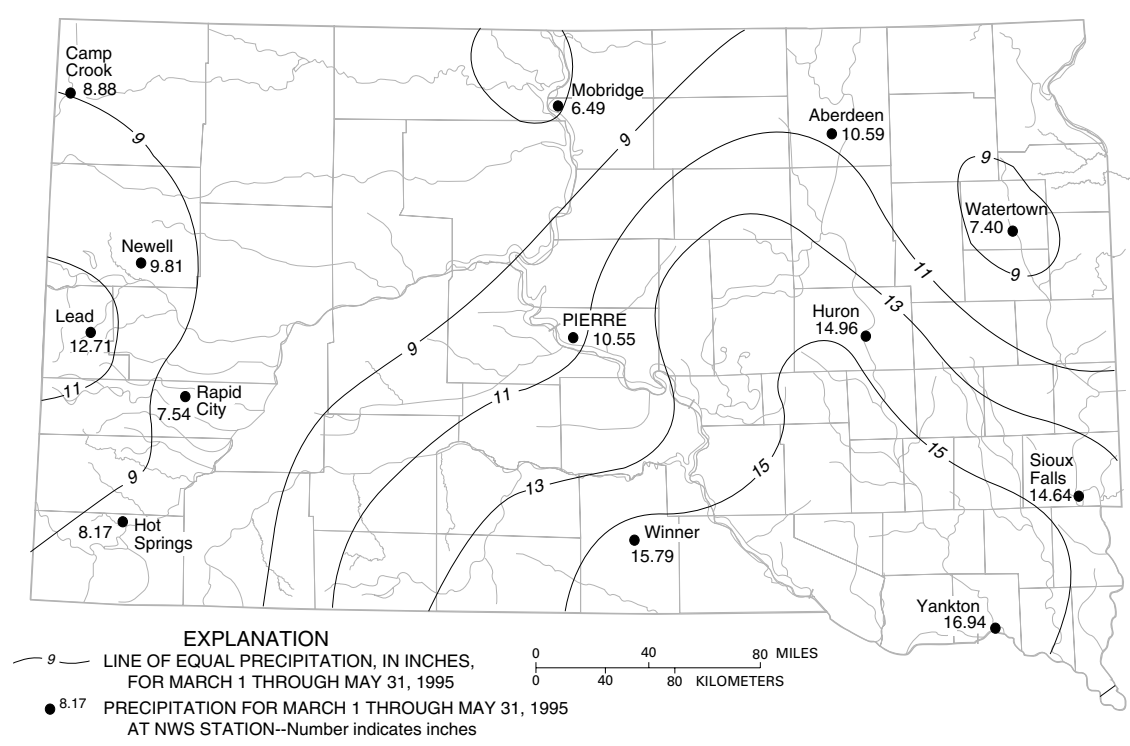

Figure 1. Preliminary National Weather Service precipitation data for March 1 through May 31, 1995 (A.R. Bender, State Climatologist, written commun., June 1995). 
Table 2. Comparison of peak stage and streamflow data for March 1 through May 31, 1995, to previous maximums at selected gaging stations for period of record

[mi ${ }^{2}$, square miles; $\mathrm{ft}$, feet; $\mathrm{ft}^{3} / \mathrm{s}$, cubic feet per second; --, no data, not computed, or not determined; <, less than; >, greater than]

\begin{tabular}{|c|c|c|c|c|c|c|c|c|c|c|}
\hline \multirow[b]{2}{*}{$\begin{array}{l}\text { Station } \\
\text { number }\end{array}$} & \multirow[b]{2}{*}{ Station name } & \multirow{2}{*}{$\begin{array}{l}\text { Period } \\
\text { of } \\
\text { record } \\
\text { (water } \\
\text { years) }\end{array}$} & \multirow[b]{2}{*}{$\begin{array}{c}\text { Contributing } \\
\text { drainage } \\
\text { area, } \\
\left(\mathrm{mi}^{2}\right)\end{array}$} & \multicolumn{4}{|c|}{ Flood of March-May 1995} & \multicolumn{3}{|c|}{ Previous maximum } \\
\hline & & & & $\begin{array}{l}\text { Peak } \\
\text { stage } \\
\text { (ft) }\end{array}$ & $\begin{array}{c}\text { Peak } \\
\text { flow } \\
\left(\mathrm{ft}^{3} / \mathrm{s}\right)\end{array}$ & Date & $\begin{array}{c}\text { Recurrence } \\
\text { interval } \\
\text { range }{ }^{1} \\
\text { (years) }\end{array}$ & $\begin{array}{c}\text { Peak } \\
\text { stage } \\
(\mathrm{ft})\end{array}$ & $\begin{array}{c}\text { Peak } \\
\text { flow } \\
\left(\mathrm{ft}^{3} / \mathrm{s}\right)\end{array}$ & Date \\
\hline 06354860 & Spring Creek near Herreid & $\begin{array}{l}1963-86 \\
1989-95\end{array}$ & 220 & 12.25 & 1,680 & $3 / 15$ & $10-25$ & 12.56 & 1,570 & $7 / 27 / 93$ \\
\hline 06354882 & Oak Creek near Wakpala & $1985-95$ & 356 & 18.58 & 5,000 & $3 / 14$ & -- & $\begin{array}{l}17.73 \\
18.35\end{array}$ & $\begin{array}{r}3,780 \\
2\end{array}$ & $\begin{array}{r}3 / 4 / 86 \\
3 / 23 / 87\end{array}$ \\
\hline 06402430 & Beaver Creek near Pringle & $1991-95$ & 45.8 & 9.10 & 60 & $5 / 7$ & -- & 8.46 & 28 & $6 / 8 / 93$ \\
\hline 06402995 & $\begin{array}{l}\text { French Creek above Stockade } \\
\text { Lake, near Custer }\end{array}$ & $1991-95$ & 68.7 & 7.40 & 375 & $5 / 8$ & $10-25$ & $\begin{array}{l}7.31 \\
--\end{array}$ & 320 & $\begin{array}{l}6 / 8 / 91 \\
8 / 6 / 91\end{array}$ \\
\hline 06403300 & French Creek above Fairburn & $1982-95$ & 105 & 3.80 & 3 & $5 / 8$ & -- & 2.73 & 329 & $3 / 7 / 87$ \\
\hline 06404800 & $\begin{array}{l}\text { Grace Coolidge Creek near } \\
\text { Hayward }\end{array}$ & 1990-95 & 7.48 & 7.62 & 378 & $5 / 8$ & -- & 7.23 & 210 & $6 / 3 / 91$ \\
\hline 06404998 & $\begin{array}{l}\text { Grace Coolidge Creek near } \\
\text { Game Lodge, near Custer }\end{array}$ & $\begin{array}{l}1972, \\
1977-95\end{array}$ & 25.2 & 10.86 & 884 & $5 / 8$ & $10-25$ & $\begin{array}{l}12.76 \\
10.84\end{array}$ & $\begin{array}{r}2-- \\
1,030\end{array}$ & $\begin{array}{l}2 / 9 / 79 \\
9 / 7 / 89\end{array}$ \\
\hline 06406000 & Battle Creek at Hermosa & $1950-95$ & 178 & 12.70 & 2,360 & $5 / 8$ & $10-25$ & 17.72 & 21,400 & $6 / 10 / 72$ \\
\hline 06406500 & Battle Creek below Hermosa & $\begin{array}{l}\text { 1951-53, } \\
1989-95\end{array}$ & 285 & 9.29 & 1,360 & $5 / 9$ & -- & $\begin{array}{l}-- \\
8.57\end{array}$ & $\begin{array}{r}2,060 \\
--\end{array}$ & $\begin{array}{r}5 / 23 / 52 \\
6 / 8 / 93\end{array}$ \\
\hline 06406920 & $\begin{array}{l}\text { Spring Creek above Sheridan } \\
\text { Lake, near Keystone }\end{array}$ & $1991-95$ & 127 & 11.84 & 651 & $5 / 8$ & -- & 10.77 & 455 & $6 / 4 / 91$ \\
\hline 06407500 & Spring Creek near Keystone & $\begin{array}{l}1946-47 \\
1987-95\end{array}$ & 163 & 7.15 & 1,100 & $5 / 9$ & $10-25$ & -- & $\begin{array}{r}865 \\
--\end{array}$ & $\begin{array}{r}6 / 23 / 47 \\
6 / 8 / 93\end{array}$ \\
\hline 06410500 & $\begin{array}{l}\text { Rapid Creek above Pactola } \\
\text { Reservoir, at Silver City }\end{array}$ & $1954-95$ & 292 & 7.88 & 839 & $5 / 8$ & $10-25$ & 10.44 & 2,060 & $5 / 15 / 65$ \\
\hline 06423010 & $\begin{array}{l}\text { Boxelder Creek near Rapid } \\
\text { City }\end{array}$ & $1978-95$ & 128 & 33.09 & 1,050 & $5 / 10$ & $<10$ & 31.65 & $\begin{array}{r}253 \\
--\end{array}$ & $\begin{array}{l}5 / 18 / 78 \\
6 / 17 / 93\end{array}$ \\
\hline 06424000 & Elk Creek near Roubaix & $\begin{array}{l}\text { 1946, } \\
1992-95\end{array}$ & 21.5 & -- & 354 & $5 / 8$ & -- & 7.87 & 154 & $6 / 8 / 93$ \\
\hline 06425100 & Elk Creek near Rapid City & $1981-95$ & 190 & 12.05 & 2,860 & $5 / 9$ & $10-25$ & $\begin{array}{l}10.79 \\
11.80\end{array}$ & $\begin{array}{r}1,560 \\
2 \\
--\end{array}$ & $\begin{array}{l}5 / 20 / 82 \\
2 / 26 / 86\end{array}$ \\
\hline 06428500 & $\begin{array}{l}\text { Belle Fourche River at } \\
\text { Wyoming-South Dakota } \\
\text { State line }\end{array}$ & $1947-95$ & 3,280 & 16.33 & 3 & $5 / 10$ & -- & $\begin{array}{l}15.59 \\
14.03\end{array}$ & $\begin{array}{l}4,400 \\
4,550\end{array}$ & $\begin{array}{l}6 / 18 / 62 \\
6 / 30 / 93\end{array}$ \\
\hline 06430500 & $\begin{array}{l}\text { Redwater Creek at Wyoming- } \\
\text { South Dakota State line }\end{array}$ & $\begin{array}{l}\text { 1929-31, } \\
1936-37 \\
1955-95\end{array}$ & 471 & 10.62 & 1,560 & $5 / 9$ & $10-25$ & 12.19 & 2,440 & $8 / 22 / 73$ \\
\hline 06430770 & Spearfish Creek near Lead & $1989-95$ & 63.5 & 8.20 & 140 & $5 / 8$ & -- & $\begin{array}{l}7.79 \\
7.64\end{array}$ & $\begin{array}{l}2-- \\
51\end{array}$ & $\begin{array}{r}1 / 29 / 91 \\
6 / 8 / 93\end{array}$ \\
\hline 06430800 & Annie Creek near Lead & 1989-95 & 3.55 & 5.94 & 3 & $5 / 8$ & -- & $\begin{array}{l}5.18 \\
4.96\end{array}$ & $\begin{array}{l}2-- \\
19\end{array}$ & $\begin{array}{r}3 / 17 / 93 \\
6 / 8 / 93\end{array}$ \\
\hline 06430850 & $\begin{array}{l}\text { Little Spearfish Creek near } \\
\text { Lead }\end{array}$ & 1989-95 & 25.8 & 5.34 & 65 & $5 / 10$ & -- & 4.95 & 22 & $4 / 25 / 94$ \\
\hline 06430898 & Squaw Creek near Spearfish & 1989-95 & 6.95 & 7.89 & 860 & $5 / 8$ & -- & 5.37 & 96 & $6 / 8 / 93$ \\
\hline 06430900 & $\begin{array}{l}\text { Spearfish Creek above } \\
\text { Spearfish }\end{array}$ & 1989-95 & 139 & 7.42 & 2,400 & $5 / 8$ & -- & 5.14 & 299 & $6 / 8 / 93$ \\
\hline 06431500 & Spearfish Creek at Spearfish & $\begin{array}{l}1904, \\
1947-95\end{array}$ & 168 & 10.70 & 2,600 & $5 / 8$ & $25-50$ & -- & 5,000 & $6 / 5 / 04$ \\
\hline 06432020 & $\begin{array}{l}\text { Spearfish Creek below } \\
\text { Spearfish }\end{array}$ & 1989-95 & 204 & 7.37 & 1,550 & $5 / 9$ & -- & $\begin{array}{l}5.86 \\
5.31\end{array}$ & $\begin{array}{r}2 \\
-- \\
163\end{array}$ & $\begin{array}{l}2 / 9 / 89 \\
6 / 6 / 91\end{array}$ \\
\hline 06433000 & $\begin{array}{l}\text { Redwater River above Belle } \\
\text { Fourche }\end{array}$ & $1946-95$ & 920 & 8.83 & 4,610 & $5 / 9$ & $10-25$ & 11.69 & 16,400 & $6 / 6 / 62$ \\
\hline 06433500 & Hay Creek at Belle Fourche & $1954-95$ & 121 & 10.23 & 1,310 & $5 / 9$ & 100 & 9.15 & 930 & $6 / 19 / 72$ \\
\hline 06436000 & $\begin{array}{l}\text { Belle Fourche River near } \\
\text { Fruitdale }\end{array}$ & $1946-95$ & 4,540 & 14.00 & 12,000 & $5 / 9$ & $10-25$ & 14.32 & 12,700 & $5 / 20 / 82$ \\
\hline 06436156 & Whitetail Creek at Lead & 1989-95 & 6.15 & 6.67 & 3 & $5 / 8$ & -- & 3.56 & 39 & $7 / 20 / 93$ \\
\hline 06436170 & $\begin{array}{l}\text { Whitewood Creek at } \\
\text { Deadwood }\end{array}$ & 1982-95 & 40.6 & 10.54 & 5,000 & $5 / 8$ & $25-50$ & 7.54 & 2,660 & $5 / 15 / 82$ \\
\hline
\end{tabular}


Table 2. Comparison of peak stage and streamflow data for March 1 through May 31, 1995, to previous maximums at selected gaging stations for period of record-Continued

\begin{tabular}{|c|c|c|c|c|c|c|c|c|c|c|}
\hline \multirow[b]{2}{*}{$\begin{array}{l}\text { Station } \\
\text { number }\end{array}$} & \multirow[b]{2}{*}{ Station name } & \multirow{2}{*}{$\begin{array}{l}\text { Period } \\
\text { of } \\
\text { record } \\
\text { (water } \\
\text { years) }\end{array}$} & \multirow[b]{2}{*}{$\begin{array}{l}\text { Contributing } \\
\text { drainage } \\
\text { area, } \\
\left(\mathrm{mi}^{2}\right)\end{array}$} & \multicolumn{4}{|c|}{ Flood of March-May 1995} & \multicolumn{3}{|c|}{ Previous maximum } \\
\hline & & & & $\begin{array}{l}\text { Peak } \\
\text { stage } \\
\text { (ft) }\end{array}$ & $\begin{array}{l}\text { Peak } \\
\text { flow } \\
\left(\mathrm{ft}^{3} / \mathrm{s}\right)\end{array}$ & Date & $\begin{array}{c}\text { Recurrence } \\
\text { interval } \\
\text { range }{ }^{1} \\
\text { (years) }\end{array}$ & $\begin{array}{l}\text { Peak } \\
\text { stage } \\
\text { (ft) }\end{array}$ & $\begin{array}{l}\text { Peak } \\
\text { flow } \\
\left(\mathrm{ft}^{3} / \mathrm{s}\right)\end{array}$ & Date \\
\hline 06436180 & $\begin{array}{l}\text { Whitewood Creek above } \\
\text { Whitewood }\end{array}$ & 1983-95 & 56.3 & 9.06 & 3 & $5 / 8$ & -- & 5.68 & 2,080 & $6 / 5 / 91$ \\
\hline 06436190 & $\begin{array}{l}\text { Whitewood Creek near } \\
\text { Whitewood }\end{array}$ & $1982-95$ & 77.4 & 6.01 & $3--$ & $5 / 8$ & -- & 4.52 & 3,050 & $5 / 20 / 82$ \\
\hline 06436198 & Whitewood Creek above Vale & $1983-95$ & 102 & 5.72 & 4,200 & $5 / 8$ & $10-25$ & $\begin{array}{l}4.32 \\
5.06\end{array}$ & $\begin{array}{l}3,680 \\
2,520\end{array}$ & $\begin{array}{r}9 / 24 / 86 \\
5 / 5 / 93\end{array}$ \\
\hline 06436760 & Horse Creek above Vale & $1981-95$ & 464 & 18.82 & 7,800 & $5 / 10$ & $10-25$ & 24.80 & 17,700 & $5 / 21 / 82$ \\
\hline 06437000 & $\begin{array}{l}\text { Belle Fourche River near } \\
\text { Sturgis }\end{array}$ & $1946-95$ & 5,870 & 17.10 & 21,500 & $5 / 10$ & $25-50$ & 19.10 & 36,400 & $5 / 21 / 82$ \\
\hline 06437020 & $\begin{array}{l}\text { Bear Butte Creek near } \\
\text { Deadwood }\end{array}$ & $1989-95$ & 16.6 & 8.85 & 2,000 & $5 / 8$ & -- & 7.70 & 938 & $6 / 5 / 91$ \\
\hline 06437500 & Bear Butte Creek near Surgis & $\begin{array}{l}\text { 1946-72, } \\
1990-95\end{array}$ & 192 & 10.96 & 5,460 & $5 / 8$ & $10-25$ & 12.45 & 12,700 & $6 / 16 / 62$ \\
\hline 06438000 & $\begin{array}{l}\text { Belle Fourche River near Elm } \\
\text { Springs }\end{array}$ & $\begin{array}{l}1929-32 \\
1934-95\end{array}$ & 7,210 & 15.29 & 29,500 & $5 / 10$ & $10-25$ & $\begin{array}{l}15.90 \\
18.22\end{array}$ & $\begin{array}{l}45,100 \\
40,300\end{array}$ & $\begin{array}{r}6 / 8 / 64 \\
5 / 21 / 82\end{array}$ \\
\hline 06438500 & $\begin{array}{l}\text { Cheyenne River near } \\
\text { Plainview }\end{array}$ & $\begin{array}{l}\text { 1951-81, } \\
1994-95\end{array}$ & 21,600 & 19.57 & 35,000 & $5 / 10$ & $10-25$ & -- & 41,700 & $5 / 26 / 57$ \\
\hline 06452320 & Platte Creek near Platte & $1989-95$ & 741 & 11.29 & 2,600 & $5 / 11$ & -- & 7.24 & 1,600 & $6 / 17 / 93$ \\
\hline 06471000 & James River at Columbia & $1946-95$ & 2,481 & $\begin{array}{l}18.50 \\
16.95\end{array}$ & ${ }^{2} 1,660$ & $\begin{array}{l}5 / 13 \\
5 / 22\end{array}$ & $<10$ & $\begin{array}{l}16.15 \\
17.11\end{array}$ & $\begin{array}{r}2,340 \\
2--\end{array}$ & $\begin{array}{r}5 / 3 / 79 \\
3 / 24 / 87\end{array}$ \\
\hline 06471200 & $\begin{array}{l}\text { Maple River at North Dakota- } \\
\text { South Dakota State line }\end{array}$ & $1957-95$ & 384 & 12.49 & ${ }^{2} 3,000$ & $3 / 16$ & $10-25$ & 16.05 & ${ }^{2} 5,930$ & $4 / 11 / 69$ \\
\hline 06471500 & Elm River at Westport & $1946-95$ & 1,049 & $\begin{array}{l}17.64 \\
16.99\end{array}$ & $\begin{array}{r}2-- \\
4,600\end{array}$ & $\begin{array}{l}3 / 16 \\
3 / 17\end{array}$ & $10-25$ & 22.11 & 12,600 & $4 / 10 / 69$ \\
\hline 06472000 & James River near Stratford & $\begin{array}{l}1950-72 \\
1977 \\
1995\end{array}$ & 4,860 & 19.86 & 3 & $5 / 18-22$ & -- & $\begin{array}{l}18.18 \\
--\end{array}$ & $\begin{array}{r}2-- \\
5,580\end{array}$ & $\begin{array}{c}4 / 19 / 69 \\
5 / 14-15 / 69\end{array}$ \\
\hline 06473000 & James River at Ashton & $1946-95$ & 5,673 & $\begin{array}{l}22.39 \\
21.21\end{array}$ & $\begin{array}{r}2-- \\
24,500\end{array}$ & $\begin{array}{l}5 / 18 \\
5 / 22\end{array}$ & $25-50$ & $\begin{array}{l}21.17 \\
20.63\end{array}$ & $\begin{array}{r}2-- \\
5,680\end{array}$ & $\begin{array}{l}4 / 13 / 69 \\
4 / 24 / 69\end{array}$ \\
\hline 06474000 & Turtle Creek near Tulare & $\begin{array}{l}\text { 1953-56, } \\
\text { 1965-81, } \\
\text { 1984-95 }\end{array}$ & 1,124 & 14.28 & 4,300 & $5 / 11$ & $10-25$ & 18.51 & ${ }^{2} 6,000$ & $4 / 5 / 69$ \\
\hline 06475000 & James River near Redfield & $1950-95$ & 9,793 & 26.26 & 9,800 & $5 / 15$ & $>100$ & 24.93 & 7,310 & $4 / 13 / 69$ \\
\hline 06476000 & James River at Huron & $\begin{array}{l}\text { 1929-32, } \\
1944-95\end{array}$ & 11,721 & 16.86 & 10,000 & $5 / 19$ & $50-100$ & 16.70 & 9,000 & $4 / 13 / 69$ \\
\hline 06476500 & Sand Creek near Alpena & $1950-95$ & 261 & 12.91 & 1,880 & $4 / 20$ & $10-25$ & $\begin{array}{l}14.10 \\
13.35\end{array}$ & 2,240 & $\begin{array}{l}3 / 28 / 50 \\
3 / 28 / 60\end{array}$ \\
\hline 06477000 & James River near Forestburg & $1950-95$ & 13,442 & $\begin{array}{l}17.26 \\
17.08\end{array}$ & $\begin{array}{r}2-- \\
13,000\end{array}$ & $\begin{array}{l}4 / 22 \\
5 / 18\end{array}$ & $25-50$ & 17.16 & 12,500 & $4 / 9 / 69$ \\
\hline 06477500 & $\begin{array}{l}\text { Firesteel Creek near } \\
\text { Mt. Vernon }\end{array}$ & $1956-95$ & 521 & 14.98 & 5,400 & $5 / 10$ & $10-25$ & $\begin{array}{l}17.12 \\
15.34\end{array}$ & $\begin{array}{r}2-- \\
6,610\end{array}$ & $\begin{array}{l}4 / 3 / 69 \\
4 / 4 / 69\end{array}$ \\
\hline 06478000 & James River near Mitchell & $\begin{array}{l}1954-58 \\
1966-72 \\
1995\end{array}$ & 14,916 & 20.43 & 16,200 & $4 / 23$ & $50-100$ & 18.32 & 13,800 & $4 / 11 / 69$ \\
\hline 06478052 & Enemy Creek near Mitchell & $\begin{array}{l}\text { 1975-87, } \\
1989-95\end{array}$ & 163 & 13.55 & 3,500 & $5 / 10$ & $10-25$ & 15.15 & 4,280 & $6 / 22 / 84$ \\
\hline 06478300 & Dry Creek near Parkston & $\begin{array}{l}\text { 1955-80, } \\
1989-95\end{array}$ & 97.2 & -- & 2,900 & $5 / 27$ & $25-50$ & -- & 4,210 & $3 / 27 / 60$ \\
\hline 06478500 & James River near Scotland & $1929-95$ & 16,505 & 19.41 & 18,200 & $5 / 29$ & $25-50$ & 20.45 & 29,400 & $6 / 23 / 84$ \\
\hline 06478513 & James River near Yankton & $1982-95$ & 16,794 & 21.46 & 20,800 & $5 / 30$ & $10-25$ & 24.34 & 26,400 & $6 / 23 / 84$ \\
\hline 06478690 & $\begin{array}{l}\text { West Fork Vermillion River } \\
\text { near Parker }\end{array}$ & $1962-95$ & 377 & 12.35 & 4,470 & $4 / 19$ & $10-25$ & 13.14 & 6,300 & $5 / 8 / 93$ \\
\hline
\end{tabular}

\footnotetext{
${ }^{1}$ Recurrence intervals generally only given for stations with 10 or more years of record through 1993.

${ }^{2}$ Stage-flow relation affected by backwater.

${ }^{3}$ Peak-flow value is not given because rating analysis and/or indirect-measurement computations were not complete when this Fact Sheet was prepared; contact USGS District office in Rapid City to check on status of these computations.
} 


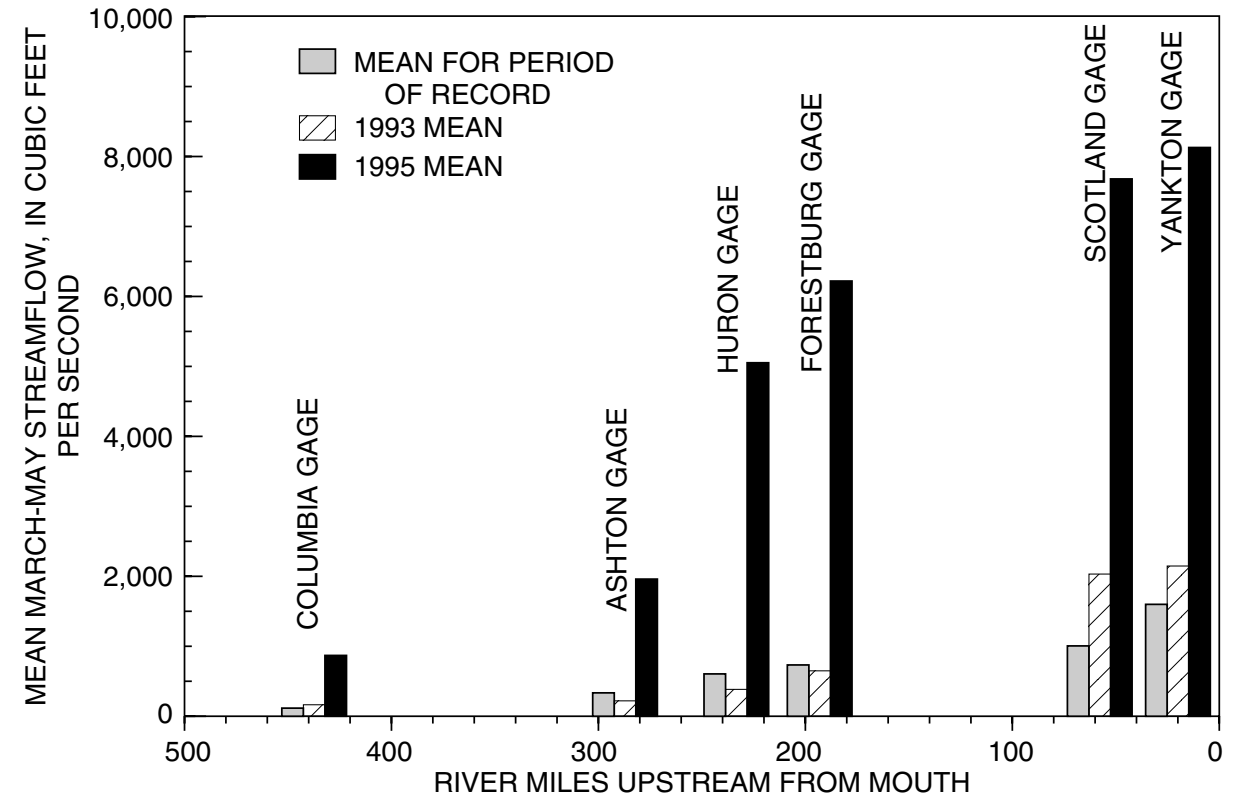

Figure 2. Mean March 1 through May 31 streamflow at James River gaging stations for period of record and 1993 and 1995.

Severe flooding occurred in western South Dakota and the Black Hills May 8-10. Flash flooding occurred across much of Butte, Custer, Lawrence, southwestern Meade, and western Pennington Counties. Damage to roads and bridges was widespread; rock and mudslides closed highways in Sturgis and in Boulder Canyon near Deadwood. Some smaller communities were isolated by road washouts. Many homes suffered water damage. About 120 Belle Fourche residents were forced from their homes on May 9 due to rising floodwaters. As the storms moved eastward, the flooding worsened in eastern South Dakota.

Streamflow on the James River was above flood stage from mid-March through May 31, 1995, and is compared in figure 2 to period-of-record and 1993 values at six gaging stations. The Missouri River reservoirs formed by the four main-stem dams in South Dakota were virtually full at the end of May and Lake Oahe was approaching a record elevation. Other lake levels in eastern South Dakota, especially in the northeast, exceeded levels that had not been reached in 100 years.

Of the 143 streamflow-gaging stations operated by the USGS in South Dakota, 34 stations that are listed in table 2 had record peak flow or stage. The other 22 stations listed in table 2 did not have record peaks, but recurrence intervals of the peaks exceeded 10 years. Several of the stations with record peaks have periods of record less than 10 years. Most of the stations listed in table 2 had peak flow with recurrence intervals between 10 and 25 years. A few stations had peak flows with recurrence intervals in the 25 to 50-year range or the 50- to 100-year range. The May 9 peak flow of $1,310 \mathrm{ft}^{3} / \mathrm{s}$ for Hay Creek at Belle Fourche had a recurrence interval equal to 100 years, and the May 15 peak flow of $9,800 \mathrm{ft}^{3} / \mathrm{s}$ for the James River near Redfield exceeded 100 years.

As a result of the severe storms and widespread flooding, 38 counties in South Dakota were approved for disaster relief by a Presidential Declaration signed on May 26. Interstate Highway 90 and many State and county highways were overtopped and subsequently closed for varying periods of time. Extensive agricultural damage occurred; for example, only 55 percent of the State's corn crop was planted as of June 4, compared to a 5-year average of 91 percent. By the end of May, the South Dakota Division of Emergency Management, working with FEMA, had conducted damage assessments in 35 counties and found that 1995 flood damage to State infrastructure already equaled the original 1993 flood-damage estimates.

\section{Monitoring Streamflow}

The USGS operates a network of 121 continuous-record streamflow-gaging stations and 22 crest-stage gages in South Dakota. The USGS also operates 47 precipitation gages in the Black Hills and one gage near Huron. During and after the flood, USGS personnel made flood measurements, indirect surveys, and surveyed high-water marks at more than 60 stations across the State.

At 31 of the streamflow stations, the data are relayed by satellite telemetry to computers in Rapid City, Huron, and Pierre (fig. 3). Data are transmitted every 15 minutes, and within 30 minutes these data generally are available to decision makers in the agencies involved in flood management. In addition, the USGS and cooperating agencies operate a network of telephone-accessible LARC and telemark gages where critical stage data are available on a real-time basis. The USGS Statewide data-collection network is readily available to local, county, State, and Federal agencies and to the public.

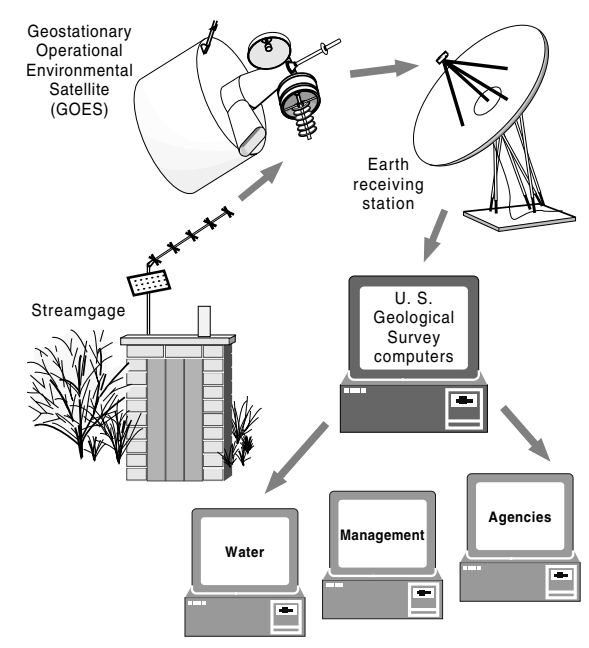

Figure 3. Schematic diagram showing how streamflow data are transmitted, processed, and distributed.

Rainfall and streamflow data in this report are provisional and subject to change upon further review by personnel of the National Weather Service and the U.S. Geological Survey.

-R.W. Teller, M. J. Burr, and D.L. Rahder

\section{For more information contact:}

District Chief

1608 Mountain View Road

Rapid City, SD 57702

(605) 394-1780 